\title{
LO BUENO, LO MALO, LO FEO Y LO NECESARIO: PASADO, PRESENTE Y FUTURO DEL REGIONALISMO LATINOAMERICANO
}

The good, the bad, the ugly and the necessary: past, present and future of Latin American regionalism

\author{
Detlef Nolte \\ German Institute of Global and Area Studies (GIGA) \\ Universidad Hamburgo \\ detlef.nolte@giga-hamburg.de \\ https://orcid.org/0000-0003-2014-272X
}

Recibido: 3/10/2018

Aceptado: 12/02/2019

\begin{abstract}
Resumen: En el contexto de la crisis actual del regionalismo latinoamericano este artículo analiza, tanto los avances como las limitaciones estructurales del regionalismo latinoamericano en los últimos 30 años. Describe algunos elementos estructurales para entender mejor la crisis del regionalismo poshegemónico. Además discute estrategias y pasos para hacer frente a la crisis actual, y para dar nuevos impulsos al regionalismo latinoamericano. Se discuten las opciones para confrontar la porosidad de la región y para aprovechar las nuevas oportunidades de establecer alianzas de cooperación económica entre países en diferentes regiones del mundo. El artículo aboga por un regionalismo pragmático y apoya la propuesta de crear un área de libre comercio latinoamericana.
\end{abstract}

Palabras clave: regionalismo latinoamericano; integración regional; regionalismo comparado. 


\begin{abstract}
In the context of the current crisis of Latin American regionalism, the first part of the article analyses both the advances and structural limitations of Latin American regionalism during the past 30 years. The second part describes some structural elements for a better understanding of the crisis of post-hegemonic regionalism. The third part discusses strategies and steps to overcome the current crisis and to give new impetus to Latin American regionalism. It discusses options how to confront the porosity of the region, and how to take advantage of the new opportunities to establish alliances for economic cooperation between countries in different world regions. The article argues in favor of pragmatic regionalism and supports the proposal to create a Latin American free trade area.
\end{abstract}

Keywords: Latin American regionalism; regional integration; comparative regionalism. 


\section{Introducción}

El título de este artículo hace referencia a una película famosa del género spaghetti western (o western europeo). Por ello el artículo no quiere decir que el regionalismo latinoamericano esté transitando de tiempos gloriosos a tiempos más complicados. Más bien, lo que quiere enfatizar, es que siempre ha habido contradicciones y tensiones en el regionalismo latinoamericano, a pesar de las diversas formas que pueda adoptar a lo largo del tiempo. Entonces, lo bueno del regionalismo latinoamericano son los factores que le han dado continuidad, así como sus avances. Lo malo, son las limitaciones estructurales - o fisurasdel regionalismo; lo feo, los desafíos y los conflictos, especialmente los de tipo coyuntural; y lo necesario (es lo nuevo de esta película), son los pasos y las estrategias que se deberían tomar para hacer frente a la crisis actual y para hacer resucitar el regionalismo latinoamericano.

El pasado del regionalismo latinoamericano no tiene fecha determinada, algunos autores lo remontan al período de la independencia. Hay antecedentes de proyectos regionales que datan de los siglos XIX y XX (Briceño-Ruiz, 2012; 2013; 2018; Paikin, Perrotta, y Porcelli, 2016; Rivarola, 2012; Rivarola y Briceño-Ruiz, 2013), especialmente desde fines de los años 1950. Pero en este artículo, cuando se habla del pasado, se refiere a los últimos 30 años. El punto de partida del estudio es la década de 1990, durante la cual los avances en el regionalismo (de la segunda ola) se manifiestan en la creación de nuevas organizaciones regionales y en la reorientación de organizaciones ya existentes. Por el presente, el artículo describe el regionalismo poshegemónico — que incluye la fase actual de paralización de proyectos regionales - ; y cuando se habla del futuro, se hace referencia a los escenarios que se presentarán para fines de esta década y el comienzo de la siguiente. 


\section{Lo bueno: una larga historia de proyectos regionales y una gran variedad de organizaciones regionales}

Existen muchos factores que han dado continuidad al regionalismo latinoamericano, los cuales justifican las expectativas actuales de que sobrevivirá y resurgirá de la crisis actual.

- Pese a ser categorizada como una región de paz violenta (Mares, 2001) y aunque persisten algunos conflictos fronterizos (Mares, 2012), América Latina ha sido una zona sin mayores conflictos bélicos. Asimismo, la región está libre de armas nucleares, y sigue una tradición legalista y de respeto al derecho internacional, basada en la existencia de un orden internacional regulado y en la resolución de disputas entre los Estados por mecanismos de la diplomacia (Kacowicz, 2005).

- Desde la independencia, ha habido varios proyectos de integración en la región. Por lo que puede decirse que América Latina destaca por la larga experiencia que ha acumulado en proyectos regionales (Briceño-Ruiz, 2018; Rivarola y Briceño-Ruiz, 2013). Esto se ha reflejado no solo en instituciones regionales, sino también en un corpus de ideas y principios de cooperación regional, así como en una comunidad epistemológica intelectual, que ha analizado y nutrido al regionalismo latinoamericano (Perrotta, 2018). El título de un artículo reciente de Alberto van Klaveren (2018), «El eterno retorno del regionalismo latinoamericano», es muy acertado.

- Ha habido una línea de continuidad de proyectos regionales durante los últimos 60 años. Existe una dependencia del trayecto (path dependency) tanto en lo bueno como en lo malo del regionalismo latinoamericano. Aunque han surgido crisis recurrentes, no ha ocurrido una ruptura definitiva. Como ejemplo están los casos del Mercado Común Centroamericano o el Pacto Andino (convertido en Comunidad Andina en 1996), que han sobrevivido etapas difíciles (como la guerra de las Cien Horas entre El Salvador y Honduras, o la salida de Chile del Pacto Andino y de Venezuela de la Comunidad Andina).

- En otras palabras, América Latina tiene la experiencia acumulada tanto de constelaciones de crisis como de la subsecuente recuperación de proyectos regionales.

- Además, varios estudios —de corte cuantitativo-demuestran que los acuerdos de comercio preferencial subregionales han impulsado la in- 
tegración regional a través de sus efectos positivos sobre el comercio interregional (Mesquita, 2018), especialmente en los años 1990.

- Cuando ha existido la necesidad y el interés de los gobiernos de la región, América Latina ha demostrado ser una región propicia y abierta para la implementación de nuevos proyectos regionales. Desde el año 2000, el número de nuevas organizaciones regionales en América Latina ha superado al de otras regiones del mundo (Börzel y Risse, 2016, pp. 624-629). Desde principios de este siglo, se han creado cuatro nuevas organizaciones regionales - ALBA, Unasur, Celac, y Alianza del Pacifico. Por tanto, podemos decir que América Latina ha sido la región más dinámica en la creación de nuevas organizaciones regionales.

- Más aún, la región se caracteriza por llevar a cabo proyectos nuevos, originales y creativos. Un ejemplo de ello es la Unasur, cuya estructura es flexible (y expandible), lo que permite que haya cooperación en diferentes áreas de políticas públicas (en sus consejos sectoriales). Otro ejemplo es la Alianza del Pacifico, que promueve la marca país (nation branding) aunque en forma colectiva (Nolte, 2016). Asimismo, tiene trece veces más países observadores que países miembros. En este aspecto constituye un caso muy particular.

- El regionalismo latinoamericano y la gobernanza regional no solo abarcan lo económico, sino también otras áreas (para el caso de Sudamérica, véase Riggirozzi y Wylde, 2018): seguridad (Mares y Kacowicz, 2016; Battaglino, 2018; Nolte y Wehner, 2014; Nolte y Weiffen, 2018), infraestructura (Palestini y Agostinis, 2015), salud (Bianculli, 2018; Herrero y Tussie, 2015; Riggirozíazi, 2015; 2014), migración (Margerithis, 2018), entre otras. Estas áreas compensan lo endeble que pueden llegar a ser las bases económicas del regionalismo latinoamericano (véase la próxima sección).

- Más importante aún, los ciudadanos latinoamericanos apoyan la integración regional. De acuerdo con la encuesta INTAL/Latinobarómetro 2017 (Basco, 2017; Beliz y Chelala, 2016), alrededor del 60 \% (2016: $60 \%$; 2017: $62 \%$ ) de los latinoamericanos está a favor de la integración política de su país con otros países de la región. Asimismo, un $77 \%$ de los ciudadanos se expresa a favor de la integración económica y del libre comercio de bienes y servicios con otros países de la región. Estos valores se han mantenido constantes entre el 2016 y el 2017, no obstante, es notable destacar que el apoyo al libre comercio aumentó (2016: $69 \%$ ). 
- Por último, vale la pena mencionar que varios actores externos apoyan los procesos de cooperación e integración regional. Dado que buscan interlocutores regionales en América Latina y el Caribe, les conviene que la integración sea entre un mayor número de países. La integración facilita el diálogo interregional, y evita el proceso de negociar con cada país latinoamericano por separado. Por ejemplo, la Unión Europea ha preferido establecer negociaciones con organizaciones regionales y subregionales; y de hecho, siempre ha apoyado a los procesos de integración en América Latina. Sin embargo, otros países, como China también han optado por establecer diálogos con los países latinoamericanos en foros multilaterales (como el foro China-Celac).

\section{Lo malo: las fisuras y limitaciones del regionalismo latinoamericano}

Las fisuras del regionalismo latinoamericano radican en los desafíos estructurales que existen para la realización de proyectos regionales. Estos desafíos o limitaciones estructurales tienen tanto una dimensión interna (inherentes a la región) como externa (ajenos a la región). Entre los desafíos más importantes se encuentran los siguientes:

- América Latina es una región fragmentada, en la que se sobreponen diferentes tipos de integración regional y subregional. Las tensiones entre los proyectos de integración latinoamericana y sudamericana son latentes y en muchos casos explícitas (Malamud C., 2015). Además, la integración de América Latina — y la del Caribe - siempre ha estado en tensión con la integración de todo el continente (esto es, las Américas, incluidos Estados Unidos y Canadá). Y el proceso de fragmentación no ha parado como hacen hincapié Quiliconi y Salgado (2017, p. 37):

[...] the current situation seems to be a construction of new regionalism through new regionalization processes that are redefining the previous regional identities and stimulating the projection of new actors in a multipolar world. It is possible to see a multiplicity of identities, namely a Pacific identity, a South American identity, a Bolivarian identity, as well as a new Latin American identity that previously seemed to be in crisis.

- Conceptos como regionalismo poshegemónico (Riggirozzi y Tussie, 2012) o regionalismo heterodoxo (Van Klaveren, 2012, p. 137) y los diversos subtipos del regionalismo latinoamericano demuestran que no existe un regionalismo latinoamericano todo-abarcador. 
- El establecimiento de límites (geográficos) a los proyectos de integración ha sido un desafío permanente en la región. A ello debe sumársele el desafío de manejar el solapamiento (overlapping) de diversas organizaciones regionales. Esto ha resultado en muchos casos en conflictos interorganizacionales y en la implementación de estrategias para debilitar o esquivar la jurisdicción de estas organizaciones regionales por parte de los países miembros (Weiffen, Wehner, y Nolte, 2013; Weiffen, 2017; Nolte, 2018).

- Aunque el comercio intrarregional ha crecido, el regionalismo latinoamericano se caracteriza por una baja regionalización económica. Los niveles de comercio intrarregional son magros en comparación con los de otras regiones (Burges, 2005; Malamud A., 2015; Defraigne, 2016; Nolte, 2017). Según cifras recientes (ECLAC, 2017), en 2016 y 2017, el comercio intrarregional latinoamericano llegó a ser el $16 \%$ del comercio total (si se excluye México, la cifra alcanza entre el 21 y el $22 \%$ ). El valor del comercio intrarregional alcanzó su valor máximo (desde 1991) en 1994, con el 21,9 \%. ${ }^{1}$ Los porcentajes son aún más bajos si solo tomamos en cuenta a los sistemas de integración subregionales (CAN 8-9 \%; Mercosur 12-13 \%; mCCA 26-28 \%; Alianza del Pacifico $3 \%)^{2}$ Las ventajas de alcanzar una mayor liberalización del comercio -objetivo central de muchos proyectos tradicionales de integración regional — han sido vistas como limitadas.

- La fragmentación de los acuerdos de comercio preferencial en América Latina en subregiones ha reforzado los efectos negativos que se presentan dado el tamaño limitado y la similitud de las ventajas comparativas de las economías involucradas (Mesquita, 2018).

- En América Latina no se han cumplido las promesas del regionalismo abierto, esto es, los acuerdos de comercio preferencial no han impulsado la competitividad de las empresas locales y sus exportaciones al mercado global. Las exportaciones extrabloque de productos del sector manufacturero no han crecido de forma significativa (Mesquita, 2018, p. 46). Además, varias estimaciones estadísticas demuestran que el

1 Esto no es nuevo. Ya los primeros estudios comparativos sobre la integración regional en América Latina, de los años 1960, hicieron hincapié al limitado tamaño del comercio. Por ejemplo, entre 1960 y 1966 el porcentaje del comercio (exportaciones) entre los miembros fundadores (Argentina, Brasil, Chile, México, Paraguay, Perú, y Uruguay) de la Asociación Latinoamericana de Libre Comercio (ALALC) aumentó de $6 \%$ a $10 \%$, y después se estancó en los dos años siguientes (Wionczek, 1970, p. 54).

2 Las estadísticas se refieren al primer semestre de 2016 y de 2017. 
margen de expansión del comercio intrarregional en América Latina y el Caribe sigue siendo bastante limitado (Mesquita, 2018). Tampoco se han creado cadenas regionales de valor como, por ejemplo, entre Argentina y Brasil en el marco del Mercosur (Viola y Lima, 2017).

- La predominancia del sector primario en las exportaciones de los países latinoamericanos ha sido un obstáculo de gran envergadura para alcanzar una mayor regionalización económica basada en sectores industriales interesados en la creación de cadenas de valor regionales (Natanson, 2017; Viola y Lima 2017). Las empresas involucradas en el comercio intraindustrial pueden llegar a convertirse en un importante grupo de presión a favor de una mayor integración regional y en contra de tendencias proteccionistas (Defraigne, 2016). En América Latina, el boom de los recursos naturales provocó — no por primera vez-un efecto contrario: disminuyó el peso del sector manufacturero en las economías. ${ }^{3}$ Como resultado: «La desunión latinoamericana es, más que cualquier otra cosa, una suma de primarizaciones nacionales» $(\mathrm{Na}-$ tanson, 2017). En la misma dirección argumentan Viola y Lima (2017) respecto a Brasil y la integración en el Mercosur. ${ }^{4}$

- Como consecuencia los países latinoamericanos tienen vínculos económicos extrarregionales más fuertes que lazos económicos intrarregionales. Un contraejemplo es la Unión Europea (pero también Asia del Este y el Pacífico) donde el porcentaje del comercio intrarregional (60\%) es mucho mayor que el porcentaje del comercio extrarregional. En total, entre 1990 y 2014, el comercio intrarregional (de todas las regiones) tuvo una participación de $45 \%$ en el comercio global. En contraste, el comercio intrarregional en América Latina y el Caribe como porcentaje del comercio total no superó el umbral del $20 \%$ (Bown, Lederman, Pienknagura, y Robertson, 2017, p. 46; IMF, 2017, p. 44).

- América Latina siempre ha sido una región porosa. Estados Unidos ha sido y todavía sigue siendo un actor importante en el regionalismo latinoamericano. La integración regional ha sido estimulada — principal-

3 Al mirar el desarrollo económico de los años 1960 Wionczek (1970, p. 61) expresa: «Paradoxically, the third major obstacle to regional cooperation arises from the improvement in the international commodity trade picture, registered in recent years under the impact of conditions of economic boom in the advanced countries. Contrary to the pessimistic ECLA predictions, the external demand for Latin America's traditional commodities improved in the 1960s».

4 We show that there has been a decrease in Brazil's trade with Mercosur, with manufactured exports being the main reason for the decline. We show that, besides the effects of China in the 2000s, there has been structural movement of Mercosur economies, mainly regarding Brazil, which supports a hypothesis of primarization or deindustrialization. (Viola y Lima, 2017, p. 3) 
mente como reacción a la influencia estadounidense- en el caso del regionalismo poshegemónico, así como bloqueada por la hegemonía estadounidense (por ejemplo, a través de la estrategia estadounidense de establecer acuerdos económicos bilaterales con países latinoamericanos después del fracaso del Acuerdo de Libre Comercio de las Américas, ALCA). Actualmente, la influencia económica de China en América Latina tiene un efecto centrífugo en el intercambio y la cooperación intrarregional. En contraste con el regionalismo europeo que responde más a incentivos intrarregionales, en el regionalismo latinoamericano la dimensión externa (como reacción a influencias e incentivos de afuera) es el motor (Riggirozzi y Tussie, 2018, p.15).

- La porosidad de la región ha provocado el surgimiento como contratendencia de la ultra defensa de la soberanía y la búsqueda de mayor autonomía (Briceño-Ruiz y Simonoff, 2015). La protección de la soberanía en contra de todo tipo de intervenciones e injerencias externas a la región ha sido un motivo importante que ha llevado a la creación de organizaciones regionales. Al respecto, autores como Deciancio (2016, p. 108) señalan que las ideas latinoamericanas sobre los proyectos de integración regional «have a long-standing history, being pioneering, both as an expression of autonomy and as way of resisting great power intervention».

- Las nociones sobre soberanía nacional —en el sentido westfaliano- ${ }^{5}$ y sobre la igualdad de los Estados están bien enraizadas en la tradición latinoamericana, así como en el principio de no intervención (Kacowicz, 2005; Serbin, 2010) La protección de la soberanía (y la independencia) habla de «una obsesión con las normas de soberanía e independencia» (Serbin, 2010, p. 8), constituye un elemento clave en el regionalismo latinoamericano.

- Se definen los empeños a favor de la protección colectiva y mutua de la soberanía de los países latinoamericanos como intentos por salvaguardar o complementar las soberanías (Ferrer, 2013); y no para juntar y compartir soberanía (lo que en inglés se denomina pooling of sovereignty). La protección de la soberanía tiene un fuerte componente dirigido hacia el exterior - es decir, en contra de las influencias ajenas a la región-, pero también está dirigido en contra de posibles injerencias

5 Por ejemplo, en el preámbulo del tratado fundacional de la Unasur se describe «que tanto la integración como la unión Sudamericana se fundan en los principios rectores de: irrestricto respeto a la soberanía $[\ldots] \gg$. 
por parte de países vecinos y de socios en los proyectos de integración regional.

- En consecuencia, la protección de la soberanía nacional «sigue constituyendo una limitación importante para la profundización de los procesos de integración regional» (Serbin, 2010, p. 18). Ciertamente, no ha habido una renuncia del derecho de veto (o de aceptar las decisiones de la mayoría) dentro de las organizaciones regionales; y se evita «cualquier clase de cesión de soberanía a entidades fuera del Estado nacional» (Levi y Reggiardo, 2016, p. 196).

- Esta orientación soberanista frena el surgimiento de una integración más profunda y la creación de instituciones regionales que sean fuertes y tengan cierto grado de independencia. La existencia de una burocracia supranacional puede dar continuidad y sustentabilidad a las actividades regionales en tiempos de crisis cuando no hay consenso entre los presidentes y existen conflictos intergubernamentales (Malamud A., 2015). Con eso no se quiere decir que organismos supranacionales son una garantía absoluta en tiempos de crisis contra la desintegración. El Brexit y la crisis de migración en Europa son contraejemplos, aumentan los costos de salida de una organización regional.

- Así, el regionalismo latinoamericano es tanto intergubernamental como interpresidencial. Esta combinación ha dado impulso a la integración regional, particularmente en épocas de fuertes liderazgos presidenciales y de afinidades políticas entre los presidentes. Sin embargo, estas características del regionalismo latinoamericano han dado lugar a bloqueos y retrasos en tiempos de polarización y de falta de consensos políticos.

- En un escenario de multipolaridad descentrada (decentred multipolarity) (Garzón, 2017) en el ámbito económico internacional, existen muchas fuerzas centrífugas que son adversas a la integración regional económica. Las interacciones económicas no están concentradas en la potencia regional de forma radial (hub-and-spoke). Más bien, se extienden fuera de la región y apuntan a todas las direcciones. Por un lado, esto hace que potencias emergentes en otras regiones puedan expandir o proyectar su influencia y sus lazos más allá de su región de referencia (por ejemplo, China hacia América Latina). Por otro lado, esto conlleva a que las potencias menores dentro de una región busquen la cooperación extrarregional para aumentar su autonomía y disminuir su dependencia con respecto a la potencia regional (Garzón, 2017, 
p. 117). Estas estrategias pueden llegar a resultar en un regionalismo cruzado en lo económico, es decir, entre países localizados en diferentes regiones (Garzón y Nolte, 2018). ${ }^{6}$ Un ejemplo de ello es la participación de tres países latinoamericanos en el Tratado Integral y Progresista de Asociación Transpacífico.

- Estos mismos incentivos sistémicos estimulan a que las potencias regionales (Brasil en el caso de Sudamérica) prescindan de las ventajas que tiene la integración regional y, por tanto, actúen de manera unilateral con el fin de maximizar los beneficios en sus relaciones con países externos a la región. Puede decirse, desde la teoría de los juegos, que estas potencias regionales se convierten en Rambos regionales, ya que prefieren adoptar estrategias de no cooperación y salirse del juego (Krapohl, Meissner y Muntschick, 2014). Un ejemplo de ello es la decisión unilateral de Brasil en 1999 de dejar flotar y depreciar la moneda nacional, lo que tuvo repercusiones negativas en otras economías del Mercosur.

- El regionalismo latinoamericano - mejor dicho, las organizaciones regionales latinoamericanas - se han caracterizado a lo largo del tiempo por incumplir los objetivos y las decisiones pactadas. Tal es el caso del Mercosur: entre 1991 y 2006 la mitad de las decisiones de su Consejo no fueron incorporados al derecho de los países miembros, debido a la falta de ratificación en las legislaturas nacionales (Malamud A., 2015).

- En América Latina hay una tendencia por externalizar la solución de controversias y conflictos - tanto comerciales como de otra índole, por ejemplo, sobre la delimitación de las fronteras- y no recurrir a los mecanismos de solución de las organizaciones o mecanismos regionales. Existe el temor de que al recurrir a estos mecanismos surja un efecto divisorio para la organización. Esto lleva a los países latinoamericanos a apelar con mayor frecuencia a organizaciones externas y neutras, como la Corte Internacional de Justicia. ${ }^{7}$ De hecho, casi la mitad de los casos que han sido remitidos a la Organización Mundial del Comercio y en los que han estado involucrados países latinoamericanos, consis-

6 "'Cross-regionalism' is the label given to the observed phenomenon of an increasing number of small-scale bilateral trade agreements between countries belonging to different regions of the world» (Garzón y Nolte, 2018, p.175).

7 En junio de 2018, cinco de los trece casos que estaban pendientes en la Corte Internacional de Justicia involucraban a países latinoamericanos. Dos casos involucraban a Chile y Bolivia, dos más a Colombia y Nicaragua, y solo un caso a Venezuela y Guyana (<http://www.icj-cij.org/en/pending-cases $>$ ). Desde 2000 a la fecha, ha habido once casos en los que han estado involucrados solamente los países latinoamericanos (<http://www.icj-cij.org/en/list-of-all-cases $>)$. 
ten en conflictos comerciales intrarregionales, que no han podido ser solucionados a través de mecanismos de arbitraje regionales (Morillo, 2017 , p. 8). ${ }^{8}$

- El regionalismo latinoamericano padece de una sobredosis de política simbólica. Un ejemplo son los parlamentos regionales que tienen pocas competencias. ${ }^{9}$ Paradójicamente, América Latina es la región del mundo con el número más alto de parlamentos regionales (Malamud C., 2015): el Parlamento Latinoamericano, el Parlamento Andino, el Parlamento Centroamericano, el Parlasur, el Parlamento Amazónico y el Parlamento Indígena. El ejemplo más ilustrativo es el del Parlamento Sudamericano que forma parte de la Unasur. Aunque a mediados de septiembre el presidente de Bolivia Evo Morales inauguró en Cochabamba el edificio que albergará a esta institución, no existió en este momento un estatuto sobre la función que tendría dicho parlamento, ni se habían designados parlamentarios. Además, la mitad de los miembros de Unasur ya no participa en las actividades de la organización.

\section{Lo feo: la crisis del regionalismo poshegemónico}

La postergación indefinida de la cumbre Celac-UE (prevista para octubre de 2017 en San Salvador), la no invitación por parte del gobierno peruano a Venezuela a la cumbre de las Américas (celebrada en Lima, en abril de 2018), y finalmente, la decisión de la mitad de los miembros de la Unasur (en ese mismo mes) de suspender su participación y los pagos hechos a la organización, son algunos de los síntomas que revelan la grave crisis del regionalismo latinoamericano. Se puede añadir la decisión del nuevo gobierno de Colombia, en agosto de 2018, de salir de Unasur, como también el anuncio del gobierno de Ecuador de destinar el edificio de Unasur a otra tarea y de salir del ALBA. Existe el riesgo que se desintegre Unasur (Mijares y Nolte, 2018). Como dice Andrés Malamud (2018, p. 167) con su particular estilo: «So much have we been discussing adjectives that we have lost sight of the noun - and regionalism may have been surreptitiously receding rather than transforming».

$8 \mathrm{Al}$ respecto, vale la pena mencionar que la prescindencia de mecanismos de escape y de imposición o recompensa diferencian al régimen de comercio de la Unión Europea de otras experiencias de integración regional, como la latinoamericana. La Unión Europea no permite que contramedidas comerciales se utilicen como recompensa entre sus miembros (Phelan, 2015).

9 Andrés Malamud (2013, p.7) describe al parlamento del Mercosur de la siguiente forma: «In fact, Parlasur has no legislative competences, no oversight capacities, is not demographically proportional, and has virtually no transnational party polices». 
La crisis del regionalismo latinoamericano se debe tanto a factores coyunturales como a causas estructurales. Entre los factores estructurales, se enumeran los siguientes:

- En el siglo XXI la integración regional no ha dado un mayor impulso al comercio intrarregional. Por el contrario, el comercio intrarregional disminuyó un $26 \%$ entre 2012 y 2017 (Mesquita, 2018)

- La desconcentración del poder en el sistema internacional ha resultado tanto en una mayor autonomía regional como en una mayor autonomía nacional. Por un lado, esto ha facilitado la integración regional, pero, por el otro lado, también la ha limitado - especialmente en lo referente al surgimiento de una institucionalidad supranacional. Víctor Mijares se refiere a esta contradicción como la paradoja de la autonomía (Mijares y Nolte, 2018). El resultado son instituciones débiles que casi no ponen límites a la actuación de los Estados miembro de las organizaciones regionales. Manda el principio del consenso, que otorga un veto a cada Estado miembro. Todo esto puede dar lugar a un bloqueo de las organizaciones regionales, como fue el caso de la (no)elección del nuevo secretario general de Unasur.

- La tendencia en los procesos de integración regional es que tengan una estructura institucional cada vez más ligera. Mientras en los años 1990 la creación de instituciones supranacionales era un tema en boga en el regionalismo latinoamericano, en los primeros años del siglo XXI, el regionalismo ligero fue el concepto dominante (Sanahuja, 2008). En la década actual, la gobernanza regional se caracteriza por tener instituciones con atribuciones aún menos vinculantes. Ya no se crean parlamentos que en el pasado eran considerados como un elemento fundamental de los proyectos de integración regional. Ni el ALBA, ni la Celac, ni la Alianza del Pacifico contemplan la creación de un parlamento. Y, el parlamento de Unasur - una organización que tiene ya 10 años de vida - existe solamente en el papel (como una declaración de buenas intenciones y con un edificio vacío en Cochabamba). Asimismo, aunque las secretarías técnicas del Mercosur y la Comunidad Andina no son muy fuertes (Closa y Casini, 2016), son más fuertes que la secretaría de Unasur (que actualmente no cuenta ni con secretario general, ni con recursos). No existen secretarías técnicas en la Celac, el ALBA, o la Alianza del Pacifico.

- Como se dijo antes, la tendencia que tienen los Estados miembro por preservar lo más posible su autonomía en las organizaciones regionales 
se combina con el interpresidencialismo del regionalismo latinoamericano. La cooperación regional ha sido facilitada por la constelación de sintonía ideológica entre los gobiernos de la región en los tiempos de la marea rosa en América Latina. Después, América Latina entró en una fase en la que había mayores conflictos ideológicos entre sus presidentes, lo que resultó en un bloqueo de nuevas iniciativas regionales y en un estancamiento de los procesos de integración regional. Esto es evidente en el impacto que ha tenido la crisis venezolana en organizaciones regionales como Celac, Unasur y ALBA.

- En el presente, no existe un liderazgo lo suficientemente fuerte en América Latina (ni singular, ni compartido) que pueda promover proyectos regionales o ponga fin a la parálisis por la que atraviesan algunas de las organizaciones regionales. Con la muerte de Chávez y la transición de Lula a Dilma Rousseff, América del Sur ha carecido de un liderazgo fuerte que impulsara la cooperación regional. A partir de la destitución de Dilma Rousseff, la atención de Brasil se ha volcado hacia la esfera nacional o doméstica. Con excepción de la Alianza del Pacifico, no se ha dado el caso de un liderazgo compartido entre diferentes gobiernos.

- Se han reforzado las tendencias hacia un regionalismo cruzado (Garzón y Nolte, 2018) y hacia la promoción de acuerdos minilaterales entre países en diferentes regiones (por ejemplo, el Comprehensive and Progressive Agreement for Trans-Pacific Partnership, CPTPP o TPP11). Eso puede llegar a tener un efecto centrífugo en los procesos de integración. De hecho, la creación de la Alianza del Pacifico en 2012 provocó una reconfiguración en el regionalismo poshegemónico sudamericano (Briceño-Ruiz y Morales, 2017). Esta organización está más orientada hacia afuera que hacia adentro, como lo ilustra la categoría de Estados asociados (los candidatos son Australia, Canadá, Nueva Zelanda y Singapur). 


\section{El futuro del regionalismo latinoamericano: lo necesario y lo posible}

En un artículo publicado antes de la crisis de Celac y Unasur, Carlos Malamud (2015, p. 112) argumentaba "regional integration is in crisis and the entire region's governments - or at least the major ones- should promote a profound debate about the characteristics such a process should have». En la situación actual, ese reclamo no solo está vigente, sino también es urgente. Un estudio reciente del Banco Interamericano de Desarrollo (BID) concluye que: «Si los gobiernos de la región están realmente comprometidos en fortalecer los fundamentos políticos y económicos en favor de la integración, el tiempo lamentablemente, no está de su lado» (Mesquita, 2018, p. 136). Existe un fundamento para reactivar el regionalismo latinoamericano, pero hay que tomar en cuenta las limitaciones mencionadas anteriormente, así como también los nuevos desafíos de la coyuntura política y económica actual.

Entre los factores positivos en la coyuntura actual cabe mencionar que la ciudadanía todavía apoya ampliamente los procesos de integración regional. De igual forma, muchos actores extrarregionales, como la UE, incluso China, apuestan a que haya una mayor integración regional en América Latina. Además, todavía hay organizaciones latinoamericanas regionales que funcionan, por ejemplo, la Alianza del Pacifico o el Mercosur. En el presente, estas organizaciones son el núcleo del regionalismo latinoamericano. Otras organizaciones regionales, como la Unasur y la Celac, siguen paralizadas, pero aún no han sido disueltas. De hecho, pueden reactivarse si las circunstancias políticas lo permiten. Alternativamente el regionalismo latinoamericano se puede reconfigurar. De no solucionarse la crisis política en Venezuela, es poco probable que haya una reactivación de la Unasur; asimismo, la Celac quedaría bloqueada para muchas decisiones. Lo que implica que otras organizaciones como el Mercosur y la Alianza del Pacifico jugarán un papel más importante en el futuro a costo de organizaciones que están en declive.

También existen factores limitantes. El futuro del regionalismo latinoamericano depende, en primer lugar, de la inserción que tiene la región en la economía y la política internacional, la que a su vez determina qué tan autónoma es la región. La autonomía de América Latina es relativa. La porosidad que tiene la región no va a desaparecer, por lo tanto, el riesgo de que aumenten las fuerzas centrifugas en la región es elevado. Se puede mencionar dos influencias centrifugas: China y otros países asiáticos, y Estados Unidos.

1. China y otros países asiáticos consolidarán su posición como socios comerciales de gran importancia (en algunos casos, los socios más im- 
portantes) para América Latina. En consecuencia, es previsible que los acuerdos de libre comercio con el área Asia-Pacifico aumenten —el TPP 11 es un buen ejemplo de ello- e influyan en los flujos comerciales latinoamericanos. Los acuerdos cruzados entre regiones constituyen un elemento para reaccionar frente a los cambios en la economía global; pero este tipo de acuerdos también provocará tendencias centrífugas dentro del regionalismo latinoamericano.

2. El gobierno de Donald Trump en Estados Unidos ha delineado una agenda puramente negativa hacia América Latina (de hecho, se puede hablar de una hegemonía negativa). No obstante, Estados Unidos sigue siendo un actor económico importante para muchos países latinoamericanos. La nueva política comercial belicosa de Estados Unidos puede tener un efecto negativo en muchos países de la región. América Latina no saldrá ilesa en caso de que haya una guerra comercial entre Estados Unidos y China o Europa (o mejor dicho, con el resto del mundo). Además, existe el riesgo de que los conflictos globales entre EE. UU. y China se expandan o tengan repercusiones negativas en América Latina. En la nueva estrategia de seguridad nacional (de diciembre de 2017) del gobierno estadounidense se menciona a China (y Rusia) como una amenaza para los intereses estadounidenses en el hemisferio occidental. Al igual que en la Guerra Fría, América Latina puede llegar a convertirse nuevamente en el campo de batalla entre dos potencias extrarregionales.

No cabe duda de que América Latina tiene que confrontar su porosidad. La región debe definirse a la vez en términos exclusivos - esto es, solamente América Latina y el Caribe. Asimismo, necesita de mejores mecanismos que le permitan articular y defender sus intereses de cara al mundo. Por otro lado, la región debe aprovechar las nuevas oportunidades que un mundo multiplex (Acharya, 2014) o de multipolaridad descentrada (Garzón, 2017) le ofrece, por ejemplo, oportunidades para establecer estrategias y alianzas entre países en diferentes regiones.

- Por un lado, América Latina debe reforzar (o reanimar) a las organizaciones regionales para representar y defender la región hacia afuera. Celac y Unasur deberían jugar este rol para América Latina y Sudamérica. La alternativa son organizaciones subregionales como el Mercosur. De igual forma, los foros China-Celac y las cumbres EU-ALC se insertan en esta lógica.

- Por el otro lado, organizaciones como la Alianza del Pacifico pueden ser fundamentales para aprovechar las nuevas oportunidades ofrecidas por el regionalismo cruzado. 
- Para que toda América Latina se beneficie a través de esta nueva estrategia, es necesario que las economías de la Alianza del Pacifico formen parte de un mercado latinoamericano ampliado (o de un Tratado de Libre Comercio de América Latina y del Caribe).

Una política de integración exitosa debe combinar lo político con lo económico y lo comercial. Las críticas que se han hecho al regionalismo abierto fueron justificadas. La integración regional es más que solo comercio. Además, la base económica y comercial del regionalismo latinoamericano es muy débil. Pero, también se puede argumentar que un proyecto de integración que no toma en cuenta la dimensión comercial no es un proyecto completo. Un proyecto regional completo necesita tener a la dimensión comercial como la base sobre la que se generará una mayor regionalización económica (Nolte, 2017), que también es beneficiaria para una estrategia de integración a la economía mundial (Bown et al., 2017; IMF, 2017).

La propuesta hecha por diferentes organizaciones regionales de crear una zona de libre comercio latinoamericana (Bown et al., 2017; Powell, 2017) puede ser un camino para seguir. ${ }^{10}$ Un tratado de libre comercio para América Latina y el Caribe tendría un mercado de alrededor de USD 5 billones (cerca de $7 \%$ del PIB mundial) (Mesquita 2018, p. 81). Además, esta opción no parece ser tan difícil. De acuerdo con el Banco Mundial (Bown et al., 2017), en el 2000, los países latinoamericanos y del Caribe tenían firmado acuerdos comerciales preferenciales con, en promedio, cuatro socios en la región. Hasta el 2013, el número promedio de acuerdos por país aumentó a diez. De igual forma, el Banco Inteamericano (Powell, 2017) señala que existen 33 acuerdos comerciales entre los Estados miembro de la región y que alrededor del $85-90 \%$ del comercio intrarregional se lleva a cabo en términos preferenciales (Mesquita, 2018). Además, se han establecido más reducciones de aranceles para el futuro; y se espera que la mayoría de los acuerdos preferenciales subregionales alcancen su potencial máximo de liberalización comercial en el año 2020 (Estevadeordal, Giordano y Ramos, 2016). En suma, hay que recoger las cosechas de los avances ya logrados.

Dicho esto, existe una brecha importante en los acuerdos de comercio preferenciales en la región. No hay un acuerdo entre México y los países del Mercosur que pueda vincular a las dos economías más importantes latinoamericanas. Esto

10 Mesquita (2018, p. 134) hace alusión a estas alternativas de la siguiente forma: «En una economía mundial que está cada vez más dominada por megaeconomías y megaacuerdos, pero que también enfrenta el desafío de una reacción proteccionista, el mosaico de pequeños ACP intrarregionales de ALC le deja pocas alternativas a la región: o bien estos ACP adquieren una masa crítica económica o enfrentarán una muerte lenta o, lo que es peor, se tornarán irrelevantes. Si los gobiernos de ALC quieren aferrarse a sus objetivos de integración regional, la respuesta es la convergencia». 
es importante, considerando que tanto México como Brasil exportan productos de mayor valor agregado y podrían facilitar la formación de cadenas de valor regionales (Mesquita, 2018, p. 34). Un acuerdo de libre comercio entre los países de la Alianza del Pacifico y del Mercosur sería un importante primer paso para cerrar la brecha en las redes de acuerdos de comercio preferencial.

Aunque el impacto inmediato de un área de libre comercio latinoamericano y del Caribe para el comercio intrarregional (en su conjunto) no es tan alto (con un aumento promedio del 3,5\%), el aumento que se puede dar en los mercados de bienes intermedios es mayor (alrededor del $9 \%$ ). Además, en caso de que haya mayores fricciones en el comercio mundial, un tratado de libre comercio latinoamericano puede mitigar los efectos negativos que habría sobre las exportaciones de los países de América Latina y el Caribe, hasta en un 40 \% (Mesquita, 2018).

Lo más importante ahora es que se eliminen las barreras no arancelarias y se amplíen y acumulen las reglas de origen entre los acuerdos existentes. Esto puede sentar las bases para crear cadenas regionales de valor. Actualmente existen 47 reglas de origen contempladas en 33 acuerdos comerciales de preferencia (Mesquita, 2018, p. 34). Además, se puede facilitar el comercio a través de la armonización de las normas técnicas y fitosanitarios; y la reducción de los costos de transporte dentro de América Latina.

La crisis de Unasur y la reorientación de la política exterior mexicana abre nuevamente el debate sobre cuáles son los objetivos de la integración: ¿la integración sudamericana o la integración latinoamericana? Unasur fue un proyecto regional que tenía un liderazgo regional claro en Brasil. Se trataba del modelo clásico de cooperación regional: una potencia regional promueve y lidera un proyecto regional (Mattli, 1999). No obstante, Brasil nunca cumplió de manera perfecta con esta función: No cargó — como paymaster - con la mayor parte del proyecto de integración regional en Sudamérica, ni tampoco ejerció un liderazgo institucional fuerte (Mouron y Onuki, 2015). De hecho, no había el apoyo domestico suficiente para que ejerciera el rol de líder regional; tampoco los brasileros se identificaban mucho con la región (Onuki, Mouron y Urdinez, 2016). En el futuro, los esfuerzos de integración en la región no deben depender demasiado del liderazgo de un solo país. Se necesita de un liderazgo compartido (o colectivo) - que, de cierta manera, caracteriza a la Alianza del Pacifico (Chaves García, 2018).

En el futuro se necesita un regionalismo pragmático y flexible (Chaves García, 2018 en referencia a la Alianza del Pacifico) tanto hacia adentro como hacia afuera. Un regionalismo pragmático y flexible: 
- Está basado en organizaciones regionales existentes, las refuerza, y trata de crear vínculos (y sintonía) entre ellas.

- Define a las políticas de integración como políticas de Estado - por ejemplo, es el caso de los miembros de la Alianza del Pacifico (Chaves García, 2018) - y no tanto como políticas cortoplacistas de los gobiernos de turno (Malamud C. 2015, p. 113).

- Acepta que las limitaciones comerciales justifiquen o hagan necesario que la cooperación regional se amplíe y cubra varios sectores de políticas públicas.

- Reconoce que no se puede desatender la dimensión comercial, ya que los lazos comerciales pueden crear vínculos (e intereses) transfronterizos fuertes.

- Hace frente a la porosidad de la región a través del reforzamiento de los vínculos intrarregionales; y aprovecha las posibilidades de interconexión entre países en diferentes regiones en el marco de un regionalismo cruzado.

- Está fundamentado en que no todos los países de la región tienen que participar en todas y cada una de las iniciativas de integración regional. De esta manera, se evitan los bloqueos y los compromisos con un denominador común bajo.

- Posee flexibilidad institucional y se basa en una geometría variable (Hofmann y Mérand, 2012) que le permite tener velocidades diferenciadas de cooperación (Muñoz, 2014).

- Reconoce que la integración económica necesita de una base latinoamericana, que incluya a México como la segunda economía regional y como la economía que está más integrada a las cadenas globales de valor. Brasil y México podrían ser como Alemania y Francia en Europa y constituir el eje del proceso de integración latinoamericano (Malamud C., 2015). Tanto el proyecto de integración sudamericano de Brasil, como el proyecto mexicano de apostar todo en el mercado norteamericano, parecen ser callejones sin salida.

- Reconoce que la gobernanza necesita de una base institucional que no necesariamente imite a la institucionalidad europea. Gobernanza regional es un proceso a través del cual se generan y establecen reglas y normas en diferentes áreas de políticas públicas. Sin embargo, una gobernanza regional que sea eficiente debe incluir también mecanismos que puedan hacer que estas normas y reglas prevalezcan. 
En lo que se refiere a la futura institucionalidad del regionalismo latinoamericano, hay que tomar en cuenta las limitaciones basadas en las trayectorias históricas (por ejemplo el alto valor que se atribuye a la defensa de la autonomía y soberanía), pero también las experiencias positivas y negativas con diferentes proyectos regionales, así como las exigencias y limitaciones de la economía-política internacional.

Hay un consenso con respecto a una institucionalidad regional ligera que no requiere de instituciones supranacionales. El Informe Macroeconómico de América Latina y el Caribe de 2017 del Banco Interamericano sintetiza, de alguna forma, esta nueva corriente dominante en el pensamiento latinoamericano:

Si algo se puede aprender en más de medio siglo de integración, es que una arquitectura compleja, como una unión aduanera con instituciones supranacionales debería evitarse. El objetivo debería ser una zona de libre comercio básica (plain vanilla) que se concentre en bienes y servicios. Con el tiempo, pueden contemplarse otros elementos [...] Esta arquitectura institucional debe tener fundamentalmente un carácter de tipo intergubernamental más que supranacional, con una comisión compuesta de ministros o funcionarios de alto nivel que supervisen la implementación y el funcionamiento del acuerdo, y orienten su evolución futura. (Powell, 2017, p. 71-74)

Hay que incluir algunos de los elementos que han resultado ser los más exitosos en las organizaciones regionales existentes, como la estructura sectorial de la Unasur; o las condiciones que impone la Alianza del Pacifico en materia de afiliación a la organización - exige el cumplimiento estricto con los criterios de acceso antes de otorgar el estatus de miembro pleno. Esta combinación parece ser prometedora. Puede ser una estrategia para conformar un núcleo de países en los que haya un consenso básico amplio sobre los objetivos de la cooperación regional y haya una cierta sintonía en políticas socioeconómicas. Esto es, los nuevos miembros que se unan a la organización en el futuro deberán cumplir con las exigencias y atribuciones básicas de la organización. Sería un procedimiento similar al acceso a la Unión Europea. Lo que se necesita son reglas claras en lo que se refiere al acceso y a la membresía de las organizaciones regionales. Desde esta perspectiva el Mercosur y la Alianza del Pacifico se pueden convertir en los ejes de la integración latinoamericana (con lazos con las organizaciones regionales de Centroamérica y del Caribe). Hay que ver si Unasur se recupera. Tal vez es más probable que se reanimen algunos consejos sectoriales (que han funcionado), siempre y cuando exista la necesidad o el interés de cooperación sectorial (plus vínculos con actores y redes sociales que promuevan esta cooperación).

Ante la crisis que enfrentan hoy en día la Unasur y la Celac, es necesario reabrir el debate y la discusión sobre las tensiones entre los principios de no 
intervención y la defensa de la soberanía, así como la necesidad de que haya un consenso sobre las normas básicas entre una comunidad de Estados. Se debe recrear o generar un nuevo consenso sobre cuáles son los valores democráticos de América Latina - esto no representa un problema solo para Venezuela, sino también para otros países. Asimismo, hay que generar y fortalecer a las instituciones regionales que protejan y promuevan la democracia y los derechos humanos. De igual forma, el regionalismo latinoamericano necesita de mejores instituciones de arbitraje e incluso tribunales regionales.

\section{Bibliografía}

Acharya, A. (2014). The End of the American World Order. Cambridge, Reino Unido: Polity Press.

BASCO, A. I. (2017). La tecno-integración de América Latina Instituciones, comercio exponencial y equidad en la era de los algoritmos. Nota Técnica IDB-TN-1340. Buenos Aires, Argentina: INTAL/Latinobarómetro, BID.

Battaglino, J. (2018). Governing security in South America: From the Inter-American treaty of reciprocal Assistance to the South American Defence Council. En P. Riggirozzi, y C. Wylde (Eds.), Handbook of South American Governance (pp. 99-110). Londres y Nueva York, Reino Unido y EE. UU.: Routledge.

Beliz, G., y Chelala, S. (2016). The DNa of Regional Integration. Latin Americans' Views on High-Quality Convergence: Innovation, Equality, and Care for the Environment. Buenos Aires, Argentina: INTAL/Latinobarómetro.

Bianculli, A. C. (2018). From free market to social policies? Mapping regulatory cooperation in education and health in Mercosur. Global Social Policy, 18(3), 249-266, doi: <https://doi.org/10.1177/1468018118780085 >.

Bown, C. P., Lederman, D., Pienknagura S., y Robertson, R. (2017). Better Neighbors: Toward a Renewal of Economic Integration in Latin America. Washington, DC, EE. UU.: World Bank.

Börzel, T. A., y Risse, T. (2016). Three Cheers for Comparative Regionalism. En T. A. Börzel, y T. Risse (Eds.), The Oxford Handbook of Comparative Regionalism (pp. 621-647). Oxford, Reino Unido: Oxford University Press.

BRICEÑo-RuIz, J. (2012). Autonomía y desarrollo en el pensamiento integracionista latinoamericano. En J. Briceño-Ruiz, A. Rivarola Puntigliano P., y Á. M. Casas Gragea (Eds.), Integración latinoamericana y caribeña (pp. 27-58). Madrid, España: Fondo de Cultura Económica. 
BriceÑo-Ruiz, J. (2013). The Andes: a long tradition of thought and practice about regional integration. En A. Rivarola Puntigliano y J. Briceño-Ruiz (Eds.), The Resilience of Regionalism in Latin America and the Caribbean. Development and Autonomy (pp. 146-182). Basingstoke, Reino Unido: Palgrave.

BRICEÑo-Ruiz, J., y Simonoff, A. (Eds.) (2015). Integración regional y cooperación en América Latina. Una relectura a partir de la teoría de la autonomía. Buenos Aires, Argentina: Editorial Biblos.

Briceño-Ruiz, J., y Morales, I. (2017). Post-Hegemonic Regionalism in the Americas. Toward a Pacific-Atlantic Divide? Londres y Nueva York, Reino Unido y EE. UU.: Routledge.

BRICEÑo-RuIZ, J. (2018). Las teorías de la integración regional: más allá del eurocentrismo. Bogotá, Colombia: Universidad Cooperativa.

Burges, S. W. (2005). Bounded by the Reality of Trade: Practical Limits to a South American Region. Cambridge Review of International Affairs, 18(3), 437454.

Chaves García, C. A. (2018). Aproximación teórica y conceptual para el análisis de la Alianza del Pacífico. Desafíos, 30(1), 21-45.

Closa, C., y Casini, L. (2016). Comparative Regional Integration. Cambridge, Reino Unido: Cambridge University Press.

DeCIANCIO, M. (2016). International Relations from the South: A Regional Research Agenda for Global IR. International Studies Review, 18(1), 106-119.

Defraigne, J. C. (2016). Is a strenghtening south-south regional integration possible? The case of Mercosur and Latin America. Fédéralisme Régionalisme, 16. Recuperado de $<$ https://bit.ly/2J0alLx $>$.

EClaC (2017). International Trade Outlook for Latin America and the Caribbean 2017: Recovery in an uncertain context. Santiago de Chile, Chile: CEPAL/ ECLAC.

Estevadeordal, A., Giordano, P., y Ramos. B. (2016). Trade and Economic Integration. En J. I. Domínguez y A. Covarrubias (Eds.), Routledge Handbook of Latin America in the World (pp.249-264). Londres y Nueva York, Reino Unido y EE. UU.: Routledge.

Ferrer, A. (27 de enero de 2013). Transformaciones de América Latina. Página 12. Recuperado de $<$ https://bit.ly/2LgUoCl $>$.

GARZón, J. F. (2017). Multipolarity and the future of economic regionalism. International Theory, 9(1), 101-135. 
Garzón, J., y Nolte, D. (2018). The new minilateralism in regional economic governance: Crossregionalism and the Pacific Alliance. En P. Riggirozzi y C. Wylde (Eds.), Handbook of South American Governance (pp. 173-189). Londres y Nueva York, Reino Unido y EE. UU.: Routledge.

Herrero, M. B., y Tussie, D. (2015). Unasur Health: A quiet revolution in health diplomacy in South America. Global Social Policy, 15(3), 261-277.

Hofmann, S., y MÉRAnd, F. (2012). Regional Organizations à la Carte: the Effects of Institutional Elasticity. En T. V. Paul (Ed.), International Relations Theory and Regional Transformation (pp. 133-157). Cambridge, Reino Unido: Cambridge University Press.

International Monetary Fund (IMF) (2017). Cluster Report - Trade Integration in Latin America and the Caribbean. IMF Country Report, 17/66. Washington, DC, EE. UU.: IMF.

Kacowicz, A. M. (2005). The Impact of Norms in International Society. The Latin American Experience, 1881-2001. Notre Dame, EE. UU.: University of Notre Dame Press.

Krapohl, S., Meissner, K., y Muntschick, J. (2014). Regional Powers as Leaders or Rambos? The Ambivalent Behaviour of Brazil and South Africa in Regional Economic Integration. Journal of Common Market Studies, 52(4), 879-895.

Leví Coral, M., y Reggiardo, G. (2016). La Alianza del Pacífico en el regionalismo sudamericano actual. Revista Mexicana de Política Exterior, 106, 187-204.

Malamud, A (2005). Presidential Diplomacy and the Institutional Underpinnings of Mercosur. An Empirical Examination. Latin American Research Review, 40(1), 138-64.

Malamud, A. (2013). Overlapping Regionalism, No Integration: Conceptual Issues and the Latin American Experiences. EUI Working Paper RSCAS 2013/20. Florence, Italia: EUI.

Malamud, A. (2015). Interdependence, Leadership and Institutionalization: The Triple Deficit and Fading Prospects of Mercosur. En S. Dosenrode (Ed.), Limits to Regional Integration (pp. 163-178). Farnham, Reino Unido: Ashgate.

Malamud, A. (2018). Book Review: Regionalism with Adjectives in Latin America. Latin American Policy, 9(1), 164-168.

Malamud, C. (2015). Regional Integration and Cooperation in Latin America: diagnosis and Proposals. Global Journal of Emerging Market Economies, 7(2), 92-120. 
Mares, D. R. (2001). Violent Peace. Militarized Interstate Bargaining in Latin America. Nueva York, EE. UU.: Columbia University Press.

Mares, D. R. (2012). Latin America and the Illusion of Peace. Londres, Reino Unido: IISS.

Mares, D. R., y KaCOWICZ, A. M. (Eds.) (2016). Routledge Handbook of Latin American Security. Londres y Nueva York, Reino Unido y EE. UU.: Routledge.

Margheritis, A. (2018). International migration in South America: Emerging forms of governance. En P. Riggirozzi y C. Wylde (Eds.), Handbook of South American Governance (pp. 415-427). Londres y Nueva York, Reino Unido y EE. UU.: Routledge.

Mattli, W. (1999). The Logic of regional Integration. Europe and Beyond. Cambridge: Reino Unido: Cambridge University Press.

Mesquita, M. (Coord.) (2018). Conectando los puntos. Una hoja de ruta para una mejor integración de América Latina y el Caribe. Washington D.C., EE. UU.: BID.

Mijares, V., y Nolte, D. (2018). Regionalismo poshegemónico en crisis ¿Por qué la Unasur se desintegra? Foreign Affairs Latinoamérica, 18(3), 105-112.

Morillo, J. (2017). WTo Dispute Settlement System: Latin American countries behavior in the period 1995-2017. Working Paper n. ${ }^{\circ}$ 21/2017. Recuperado de $<$ https://bit.ly/2H46flx>.

Mouron, F., y Onuki, J. (2015). ¿Brasil es un líder en América del Sur? El papel brasileiro a través del concepto de liderazgo situacional. Estudos Internacionais, 3(1), 9-25.

MuÑoz, H. (12 de marzo de 2014). Convergencia en la diversidad: la nueva política latinoamericana de Chile. El País. Recuperado de <https://bit.ly/11ZkeGL>.

Natanson, J. (febrero de 2017). La integración es un elefante blanco. Le Monde diplomatique. Recuperado de $<$ https://bit.ly/2VD0d0Z $>$.

Nolte, D. (2016). The Pacific Alliance: Nation-Branding through Regional Organisations. GIGA Focus Latin America 04/2016.

Nolte, D. (2017). Trade: The Undervalued Driver of Regional Integration in Latin America. GIGA Focus Latin America, 05/2017. Recuperado de <https://bit. ly/2w5YNfx>.

Nolte, D. (2018). Costs and Benefits of Overlapping Regional Organizations in Latin America: The Case of OAS and Unasur. Latin American Politics and Society, 60(1), 128-153. 
Nolte, D., y Wehner, L. (2014). Unasur and Security in South America. En S. Aris y A. Wenger (Eds.), Regional Organisations and Security. Conceptions and practices (pp. 183-202). Londres y Nueva York, Reino Unido y EE. UU.: Routledge.

Nolte, D., y Weiffen, B. (2018). Competing Claims for Security Governance in South America. En S. Aris, A. Snetkov, y A. Wenger (Eds.), Inter-organisational Relations in International Security: Cooperation and Competition (pp. 109-126). Londres y Nueva York, Reino Unido y EE. UU.: Routledge.

Onuki, J., Mouron, F., y Urdinez, F. (2016). Latin American Perceptions of Regional Identity and Leadership in Comparative Perspective. Contexto Internacional, 38(1), 433-465.

Paikin, D., Perrotta, D., y Porcelli, E. (2016). Pensamiento latinoamericano para la integración. Crítica y Emancipación, 8(15), 49-80.

Palestini, S., y Agostinis, G. (2015). Constructing regionalism in South America: the cases of sectoral cooperation on transport infrastructure and energy. Journal of International Relations and Development, 21(1), 1-29.

Perrotta, D. (2018). El campo de estudios de la integración regional y su aporte a las Relaciones Internacionales: una mirada desde América Latina. Relaciones Internacionales, 38, 9-39.

Phelan, W. (2015). Enforcement and Escape in the Andean Community: Why the Andean Community of Nations is Not a Replica of the European Union. Journal of Common Market Studies, 53(4), 840-856.

Powell, A. (2017). Caminos para crecer en un nuevo mundo comercial. Informe Macroeconómico de América Latina y el Caribe de 2017. Washington D.C., EE. UU.: Banco Interamericano de Desarrollo.

Quiliconi, C., y SALGAdo EsPinOzA, R. (2017). Latin American Integration: Regionalism à la Carte in a Multipolar World? Colombia Internacional, 92, 15-41.

RigGirozZI, P. (2014). Regionalism trough social policy: collective action and health diplomacy in South America. Economy and Society, 43(3), 432-454.

RiggirozzI, P. (2015). Regionalism, activism, and rights: new opportunities for health diplomacy in South America. Review of International Studies, 41(2), 407-428.

Riggirozzi, P., y Tussie, D. (Eds.) (2012). The Rise of Post-Hegemonic Regionalism. Dordrecht, Holanda: Springer Netherlands.

Riggirozzi, P., y Tussie, D. (Eds.) (2018). Claves para leer al regionalismo sudamericano. Fortaleciendo el estado, regulando el mercado, gestionando autonomía. Perspectivas Revista de Ciencias Sociales, 3(5), 6-21. 
Riggirozzi, P., y Grugel, J. (2015). Regional governance and legitimacy in South America: the meaning of Unasur. International Affairs, 91(4), 781-797.

Riggirozzi, P., y WyLde, C. (Eds.) (2018). Handbook of South American Governance. Londres y Nueva York, Reino Unido y EE. UU.: Routledge.

Rivarola, A. (2012). Tres vertientes del pensamiento integracionista en Latinoamérica. En J. Briceño-Ruiz, A. Rivarola Puntigliano, y À. M. Casas Gragea (Eds.), Integración latinoamericana y caribeña (pp. 79-102). Madrid, España: Fondo de Cultura Económica.

Rivarola, A., y BriceÑo-Ruiz, J. (Eds.) (2013). The Resilience of Regionalism in Latin America and the Caribbean. Development and Autonomy. Basingstoke, Reino Unido: Palgrave.

Sanahuja, J. A. (2008). Del regionalismo abierto al regionalismo posliberal. Crisis y cambio en la integración regional en América Latina. En L. Martínez, A. Lázaro Peña, M. Vázquez (Coord.), Anuario de la Integración Regional de América Latina y el Gran Caribe n. ${ }^{\circ}$ 7, año 2008-2009 (pp.11-54). Buenos Aires, Argentina: CRIES.

Serbin, A. (2010). Regionalismo y soberanía nacional en América Latina: Los nuevos desafios. Documentos CRIES 15. Buenos Aires, Argentina: CRIES.

VAN KlaVEREN, A. (2012). América Latina en un nuevo mundo. Revista CIDOB d'Afers Internacionals 100, 131-150.

VAN KlaVEREN, A. (2018). El eterno retorno del regionalismo latinoamericano. Nueva Sociedad 275, 62-72.

Viola, E., y Santos Lima, J. (2017). Divergences Between New Patterns of Global Trade and Brazil/Mercosur. Brazilian Political Science Review, 11(3), 1-31.

WeIfFen, B. (2017). Institutional Overlap and Responses to Political Crisis in South America. En M. A. G. Suarez, R. Duarte Villa, y B. Weiffen (Eds.), Power Dynamics and Regional Security in Latin America (pp. 173-199). Basingstoke, Reino Unido: Palgrave Macmillan.

WeIfFen, B., WeHner, L., y Nolte, D. (2013). Overlapping regional security institutions in South America: The case of OAS and Unasur. International Area Studies Review, 16(4), 370-389.

WionczeK, M. S. (1970). The Rise and decline of Latin American Economic Integration. Journal of Common Market Studies, 9(1), 49-66. 